\title{
AVALIAÇÃO DO COMPORTAMENTO MECÂNICO DO AÇO SAE 4140
}

\author{
Ednildo Turatti ${ }^{1}$, Gabriel Birck², Marcelo André Toso ${ }^{3}$
}

Resumo: Falhas de componentes são recorrentes em diversas áreas da indústria. Portanto, a correta caracterização do material empregado na fabricação de determinado produto é importante. As falhas por fadiga, que geralmente ocorrem em componentes com carregamentos cíclicos, são as mais comuns. Assim, com a realização correta de um ensaio de fadiga pode-se determinar o limite de resistência à fadiga dos materiais, possibilitando a avaliação dos resultados antes da fabricação de um componente por um determinado material, evitando a quebra inesperada de uma máquina ou componente mecânico. O objetivo deste artigo é avaliar o comportamento mecânico do aço SAE 4140 utilizando ensaios mecânicos de tração, dureza e impacto em corpos de prova em condições controladas em laboratório seguindo especificações estabelecidas por normas. Além disso, um ensaio de fadiga axial em um componente (parafuso utilizado na indústria automotiva) é realizado. Por fim, os valores obtidos são discutidos e comparados com outras referências, concluindo que as propriedades mecânicas do aço analisado são, na sua maioria, similares às referências encontradas na literatura.

Palavras-chave: Ensaio Mecânico. Propriedades mecânicas. Fadiga. SAE 4140.

\section{INTRODUÇÃO}

A incessante disputa por melhores preços, redução de custos e desperdícios são de grande importância para que a indústria continue competitiva diante de um mercado com alto nível de exigência. Por isso, a escolha do melhor material a ser empregado em um componente é fundamental. Além disso, a previsão de vida útil do componente também é importante, pois pode evitar futuras despesas a empresa, como em um recall. Portanto, a

1 Acadêmico do Curso de Engenharia Mecânica - UNIVATES. turatti05@hotmail.com

2 Doutorando em Engenharia Mecânica, Universidade Federal do Rio Grande do Sul. gabriel.birck@gmail.com

3 Doutor em Engenharia Mecânica, Professor do Instituto Federal de Santa Catarina. marcelo.toso@yahoo.com.br 
caracterização do material e o número de ciclos que um componente suporta são fundamentais, e essas informações são obtidas através de diversos ensaios mecânicos.

Segundo Dowling (1999), a maior parte dos componentes mecânicos contidos em estruturas, veículos e máquinas, geralmente sofre carregamentos cíclicos. Esses carregamentos podem ocasionar trincas microscópicas que podem se tornar macroscópicas, podendo levar à quebra do componente. A este processo dá-se o nome de fadiga. A fadiga é uma das principais causas de falhas em metais, representando cerca de $90 \%$ dos casos (CALLISTER JR., 2002). De acordo com relatório do Departamento de Comércio Norte Americano, cerca de $4 \%$ do PIB dos Estados Unidos é gasto com falhas ou fraturas em componentes utilizados em máquinas e estruturas mecânicas (NORTON, 2013).

Para a realização de ensaios mecânicos, primeiramente, necessitase fazer uma caracterização do material: análise da composição química, condições de fabricação, local que será empregado e tipos de cargas que atuam no componente. Dimensões de corpos de prova e outros fatores, inclusive a temperatura em que será realizado o ensaio, influenciam de maneira direta nos resultados obtidos. Além disso, o conhecimento do funcionamento do equipamento utilizado bem como o uso de normas adequadas para cada tipo de ensaio mecânico é necessário. Assim, para a obtenção de resultados realmente coerentes, confiáveis e satisfatórios todas as especificações devem ser observadas e seguidas. A escolha de um material ideal para utilização na indústria deve ser fundamentada em resultados obtidos através de ensaios mecânicos. O presente trabalho tem por objetivo executar os ensaios mecânicos de tração, impacto, dureza e fadiga em condições controladas em laboratório seguindo as especificações impostas pelas Normas Brasileiras (NBR), utilizando o aço SAE 4140. Isso ocorre devido à necessidade de seleção adequada do material baseado no desempenho do produto (parafuso) em estudo. Esta caracterização de propriedades mecânicas visa principalmente estimar o desempenho no período de "vida útil" do material, minimizando a possibilidade de degradação e falhas indesejáveis durante a utilização do produto.

Quanto a aspectos relacionados à fadiga dos materiais, Griza (2000) observou a interferência do torque na vida em fadiga de duas juntas parafusadas, em aço e alumínio, submetidos a carregamentos cíclicos. Utilizando parafusos, o pesquisador realizou ensaios mecânicos obtendo as curvas clássicas de Wohler. Com os resultados de ruptura e deformação obtidos, o autor conclui que valores de torque elevados acarretam uma vida em fadiga mais duradoura da junta, independente do tipo de material fixo utilizado.

Alvarenga Júnior, Mansur e Palma (2002) utilizaram uma metodologia existente para obter as curvas S-N do aço SAE 8620 com ensaios refrigerados e não refrigerados para a execução do ensaio de fadiga flexo rotativo. Para o ensaio refrigerado, utilizou-se o método Staircase. Esse método submete o corpo de prova a uma determinada tensão havendo a quebra do elemento 
diminui-se a tensão e repete-se o ensaio. Os autores iniciaram o ensaio com tensão superior a resistência a fadiga do material, até que houvesse a ruptura do corpo de prova. Em seguida, os demais corpos de prova foram ensaiados com tensões crescentes e decrescentes. Os autores observaram que os danos causados por tensões decrescentes foram maiores e com dispersões menores quando comparados aos corpos de prova submetidos a tensões crescentes. Os valores para dureza, limite de resistência à tração do material e o limite de resistência à ruptura diminuíram com o emprego de tensões crescentes. $\mathrm{O}$ limite de resistência à fadiga caiu, apresentando valores muito próximos para os dois casos. Ocorreu uma elevação na temperatura dos corpos de prova submetidos a ensaios sem refrigeração e tensões elevadas. Dessa forma os autores aconselham a utilização de refrigeração para que o comportamento dos materiais não seja influenciado pelo aquecimento do mesmo.

Em um estudo, Afzal e Fatemi (2004) analisaram e compararam em condições controladas em laboratório o comportamento à fadiga de uma biela constituída de aço forjado e metal em pó. Para os testes de resistência à fadiga, utilizou-se uma bancada com uma máquina flexo rotativa com controle de carga, para controle da tensão aplicada nos dois materiais. Em seguida, foram construídas as curvas S-N para visualização da vida prevista para cada material. Os autores concluem que o aço forjado tem maior vida útil de fadiga quando comparado ao metal em pó. Algumas comparações foram realizadas pelos autores relacionando custo $\mathrm{x}$ benefícios dos materiais com intuído de verificar qual melhor relação efetiva.

Matlock et al. (2005), apresentam em seu trabalho a importância de compreender as interações entre composição de liga de aço e de microestrutura junto com a preocupação com o processamento de superfície para melhorar a resistência à fadiga de aços aplicados na indústria automotiva. A primeira parte do trabalho baseia-se na realização dos testes de fadiga através de flexão com um aço carbono e na caracterização de diversas situações relevantes como: tamanho do grão, efeitos de outras ligas, tensões residuais. Enquanto que na segunda parte, tem-se os resultados obtidos dos ensaios em laboratório, junto com as curvas S-N para cada diferente composição utilizada para os testes. Os autores ressaltam que com os resultados das diferentes composições é possível otimizar o comportamento em fadiga dos aços de superfície modificada através de adição de outras ligas.

Maruta et al. (2013) investigaram a resistência à fadiga de amortecedores fabricados com componentes de chumbo que constitui os amortecedores de ligação em sistemas de isolamento sísmico. Para a realização dos testes foi utilizada uma mesa flexo rotativa para simular as pequenas oscilações. Os testes de fadiga foram avaliados para vida em alto ciclo com temperatura em torno de $21^{\circ} \mathrm{C}$. Foram revestidas algumas amostras com massa lubrificante e ensaiadas nas mesmas condições com o intuito de verificar se houve ganho significativo quanto comparado ao material puro. Após a realização dos testes, os autores 
observaram que houve um aumento de 5 a 10 vezes na resistência à fadiga nas amostras com revestimento quando comparadas a amostras de chumbo puro.

Abrahão et al. (2008), abordaram a relevância do estudo da falha por fadiga em materiais submetidos a ciclos repetitivos de tensão ou deformação. Os autores, descreveram os mecanismos e formato de corpos de prova usados nos ensaios de fadiga. Assim como a importância dos ensaios de fadiga em diversas aéreas. Os pesquisadores ressaltaram que o estudo da fadiga realizado em materiais é de extrema relevância para todas as áreas da engenharia e proporcionam avanços e soluções para diversas situações impostas nos processos.

Sena (2010) analisou o comportamento da fadiga em um aço bifásico DP-780 e comparou os resultados com o desempenho de aços bifásicos de alta resistência mecânica. $\mathrm{O}$ estudo averiguou o comportamento do aço sobre fadiga com ensaios de controle de tensão, com verificação da sua curva S-N e uma análise metalográfica para observar a microestrutura do material. Com o ensaio de tração, obteve-se o limite de escoamento, limite de resistência, deformação total e redução da área. Quanto ao ensaio de fadiga, submeteu-se o material a uma carga de $95 \%$ do limite de resistência, gerando assim a curva S-N. O autor conclui que a utilização do aço DP-780 é promissora, observando maior refino microestrutural, menor perda de ductilidade, limite de fadiga superior quando comparado a outros materiais bem como ganho de resistência mecânica.

Em um estudo aplicado a equipamentos térmicos, Ragagnin (2015) realizou uma análise de fadiga em uma haste de compressores alternativos. $\mathrm{O}$ autor empregou expressões analíticas para determinar os esforços atuantes na haste do compressor. Como resultado, destaca-se que mesmo havendo alteração nas condições de operação do compressor não houve sobrecarga considerável. Ressalta-se ainda, que a parte mais crítica da haste é a rosca. Portanto, concluise que a haste do projetor deve ser projetada para vida infinita.

Por fim, cita-se o trabalho de Toso et al. (2016) os autores realizaram um estudo comparativo empregando ensaios de fadiga em dois corpos de prova com geometrias diferentes, um com seção retangular e outra cilíndrica, utilizados em molas de suspensão automotiva. No ensaio experimental, as condições de teste assemelham-se mais as condições reais de uso do componente, submetidos a esforços de flexão, enquanto que para o ensaio normalizado utilizou-se o método dos elementos finitos. Assim, são comparadas as curvas S-N geradas visando identificar as causas e consequências para as diferenças encontradas na vida em fadiga nos procedimentos realizados. Através dos resultados, observou-se que com o método numérico é possível determinar o limite de resistência à fadiga do material, mas não determinar a resistência à fadiga de um componente. Os autores concluíram que o ensaio numérico é útil para definir as curvas de fadiga do material, enquanto que o ensaio experimental prevê a vida em fadiga do componente. 


\section{METODOLOGIA}

As etapas desenvolvidas nesse trabalho são apresentadas no fluxograma da Figura 1 na qual define os ensaios mecânicos a serem realizados.

Figura 1 - Fluxograma representando a metodologia utilizada no trabalho

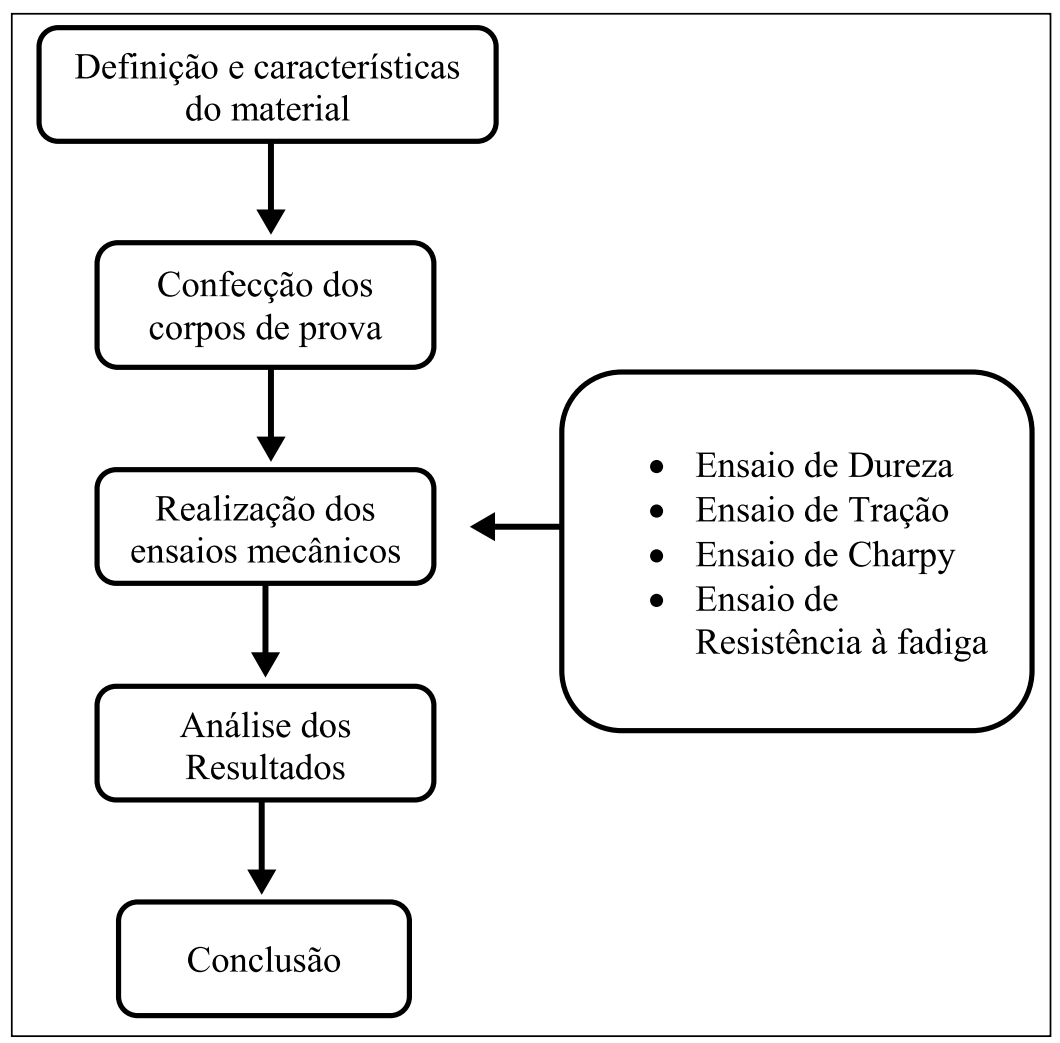

Fonte: Do autor (2016).

O material utilizado para a realização dos ensaios foi retirado de uma barra trefilada, normalizada e recozida de um aço SAE 4140, utilizado largamente na fabricação de componentes mecânicos. Conforme Chiaverini (2005) trata-se de um aço de médio carbono e baixa liga, composto por $\mathrm{Cr}$ e Mo, o que proporciona ao aço uma boa resistência com um custo relativamente baixo para a fabricação de componentes. Os valores da sua composição química são especificados pela Norma NBR NM 87 (ABNT, 2000) e estão apresentados na Tabela 1. 
Tabela 1 - Composição química do aço SAE 4140

\begin{tabular}{c|c|c|c|c|c|c|c|c|c|c}
\hline Aço & $\mathbf{C}$ & $\mathbf{S i}$ & $\mathbf{M n}$ & $\mathbf{P}$ & $\mathbf{S}$ & $\mathbf{N i}$ & $\mathbf{C r}$ & $\mathbf{M o}$ & $\mathbf{C u}$ & $\mathbf{A l}$ \\
\hline $\mathbf{4 1 4 0}$ & $0,38-0,43$ & $0,15-0,30$ & $0,75-0,90$ & 0,025 máx. & 0,025 máx. & 0,25 máx. & $0,90-1,10$ & $0,15-0,25$ & 0,05 máx. & $0,02-0,08$ \\
\hline
\end{tabular}

Fonte: Norma NBR NM 87 (ABNT, 2000, p. 9).

Para o ensaio de tração os corpos de prova foram usinados e confeccionados de acordo com a ilustração demostrada na Figura 2, seguindo as especificações da Norma NBR ISO 6892 (ABNT, 2002). Já o corpo de prova para o ensaio de impacto Charpy segue as especificações da Norma ASTM E23 (ASTM, 2002). Na Figura 3 observa-se um modelo do corpo de prova para o ensaio de impacto.

Figura 2 - Representação do corpo de prova

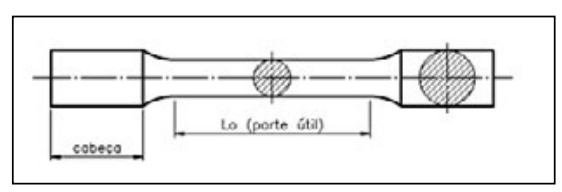

Fonte: Dalcin (2007, p. 19).

Figura 3 - Modelo do corpo de prova para ensaio de impacto

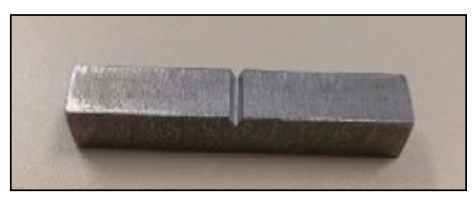

Fonte: Do autor, através do Laboratório de Engenharia Mecânica UNIVATES (2016).

Utiliza-se para o ensaio de fadiga um parafuso M8 de acordo com a norma DIN EN 1665. Desta forma, não foi necessário à confecção dos corpos de prova. Com intuito de analisar a dureza do material, são realizados testes de dureza na escala Rockwell (HR), em condições controladas em laboratório. Para este teste, utilizou-se um durômetro PANTEC, modelo RASN- RB, com penetrador de diamante e capacidade de carga de $150 \mathrm{~kg}$. A Figura 4 apresenta o durômetro previamente citado. Para estabelecer as propriedades mecânicas do material, como alongamento, estricção e limite de escoamento, quatro corpos de prova são submetidos ao ensaio de tração. Esse ensaio é realizado em uma máquina de tração da marca SHIMADZU, modelo AG-X, com capacidade de empregar forças de $250 \mathrm{KN}$. Na Figura 5 observa-se o equipamento utilizado. 
Figura 4 - Durômetro

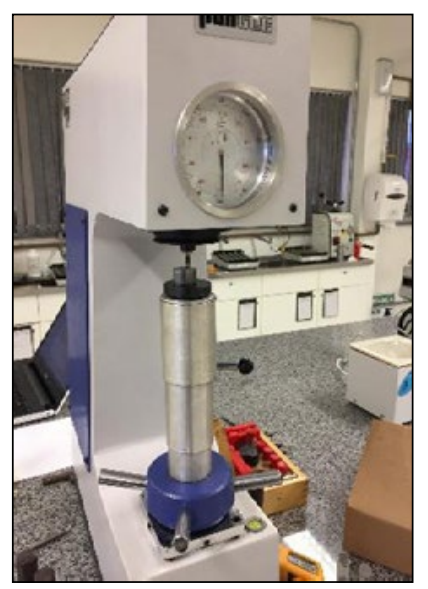

Fonte: Do autor, através do Laboratório de Engenharia Mecânica UNIVATES (2017).

Figura 5 - Máquina universal de ensaios mecânicos

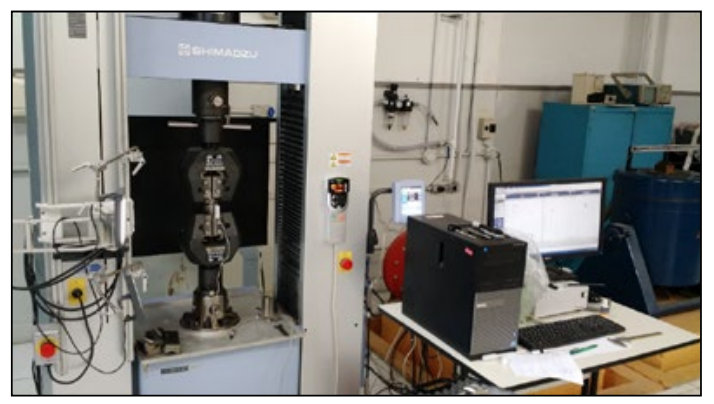

Fonte: Do autor, através do Laboratório da UFRGS (2017).

Após a realização do ensaio, com os dados obtidos calculam-se através das Equações 1 e 2 alongamento e estricção, respectivamente.

$$
\begin{aligned}
& \lambda=\left(\frac{\mathrm{Lf}-\mathrm{Li}}{\mathrm{Li}}\right) \times 100 \\
& \varphi=\left(\frac{\mathrm{Ai}-\mathrm{Af}}{\mathrm{Af}}\right) \times 100
\end{aligned}
$$

Quanto ao equipamento utilizado para os testes de fadiga (FIGURA 6), trata-se de um equipamento suíço da marca RUMUL, modelo TESTRONIC 250 kN, O ensaio aplicado é chamado de Staircase, esse método ensaia o componente a 10 milhões de ciclos. O ensaio de impacto é realizado com o objetivo de medir a capacidade do corpo de prova absorver energia. $\mathrm{O}$ equipamento é da marca PANTEC, modelo FIT-300, que pode ser visualizado na Figura 7. 
Figura 6 - Máquina para ensaio de fadiga

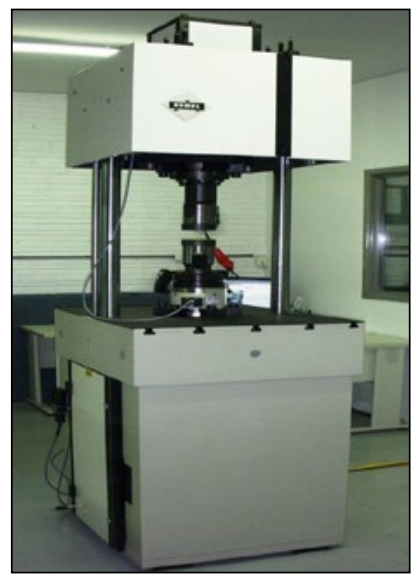

Fonte: Empresa Metalmecânica (2017, [arquivo particular]).

Figura 7 - Máquina de Charpy

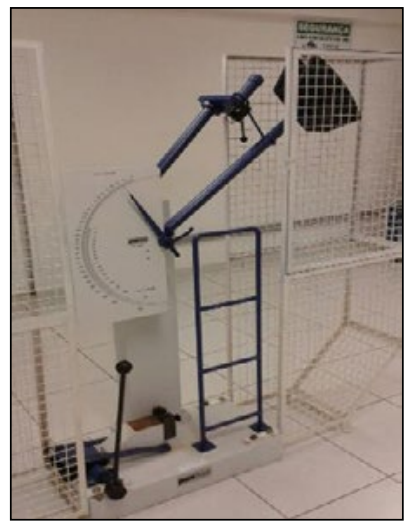

Fonte: Do autor, através do Laboratório de Engenharia Mecânica UNIVATES (2017).

Todos os corpos de prova foram submetidos à análise de falha logo após a realização dos ensaios de fadiga. Desta forma, o aspecto visual da fratura dos corpos de prova para visualizar os três estágios da fratura por fadiga foi observado. Para isso, foi utilizado um microscópico manual da marca DINOLITE, modelo AM4013 MT, ilustrado na Figura 8. 
Figura 8 - Microscópico manual

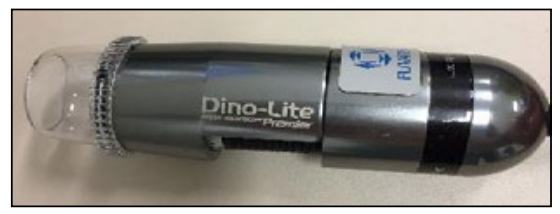

Fonte: Do autor, através do Laboratório de Engenharia Mecânica UNIVATES (2017).

\section{RESULTADOS E DISCUSSÕES}

Os resultados de dureza obtidos para o aço SAE 4140 encontram-se descritos na Tabela 2.

Tabela 2 - Valores médios de dureza de núcleo.

\begin{tabular}{|c|c|c|c|c|c|c|c|}
\hline \multicolumn{8}{|c|}{ Dureza de Núcleo Encontrada (Rockwell) } \\
\hline & \multicolumn{5}{|c|}{$\mathrm{N}^{\circ}$ do ensaio } & Valor médio & Desvio padrão \\
\hline Amostras & 1 & 2 & 3 & 4 & 5 & (HRc) & \\
\hline 1 & 27 & 28 & 27 & 28 & 27 & 27,4 & 0,49 \\
\hline 2 & 27 & 31 & 28 & 26 & 28 & 28,0 & 1,67 \\
\hline 3 & 26 & 26 & 27 & 27 & 28 & 26,8 & 0,75 \\
\hline 4 & 27 & 28 & 29 & 30 & 29 & 28,6 & 1,02 \\
\hline
\end{tabular}

Fonte: Do autor (2017).

Através da Tabela 3 observa-se que os resultados obtidos do aço SAE 4140 encontra-se na faixa estabelecida pela norma DIN 10083 (EUROPEAN STANDARD, 2006) para temperatura $25^{\circ} \mathrm{C}$, os quais são de 26 à $42 \mathrm{HRc}$. A Tabela 3 apresenta os valores obtidos para resistência à tração e escoamento do material.

Tabela 3 - Valores de resistência à tração e de escoamento para o aço 4140.

\begin{tabular}{c|c|c|c|c|c|c}
\hline \multicolumn{7}{c}{ Resistência à tração e escoamento do aço 4140 (MPa) } \\
\hline Amostras & $\mathbf{1}$ & $\mathbf{2}$ & $\mathbf{3}$ & $\mathbf{4}$ & $\begin{array}{c}\text { Valor } \\
\text { médio }\end{array}$ & $\begin{array}{c}\text { Desvio } \\
\text { padrão }\end{array}$ \\
\hline Resistência à tração & 937,91 & 944,73 & 934,18 & 918,17 & 933,75 & 11,27 \\
\hline $\begin{array}{c}\text { Resistência ao } \\
\text { escoamento }\end{array}$ & 661,34 & 658,43 & 661,63 & 664,91 & 661,58 & 2,65 \\
\hline $\begin{array}{c}\text { Área do corpo de prova } \\
\left(\mathbf{m m}^{2}\right)\end{array}$ & 31,172 & 31,172 & 31,172 & 30,484 & 31,00 & 0,34 \\
\hline
\end{tabular}

Fonte: Doa autor (2017). 
A tensão versus deformação para os quatro corpos de prova analisados é apresentada na Figura 9.

Figura 9 - Gráfico tensão x deformação para o aço 4140

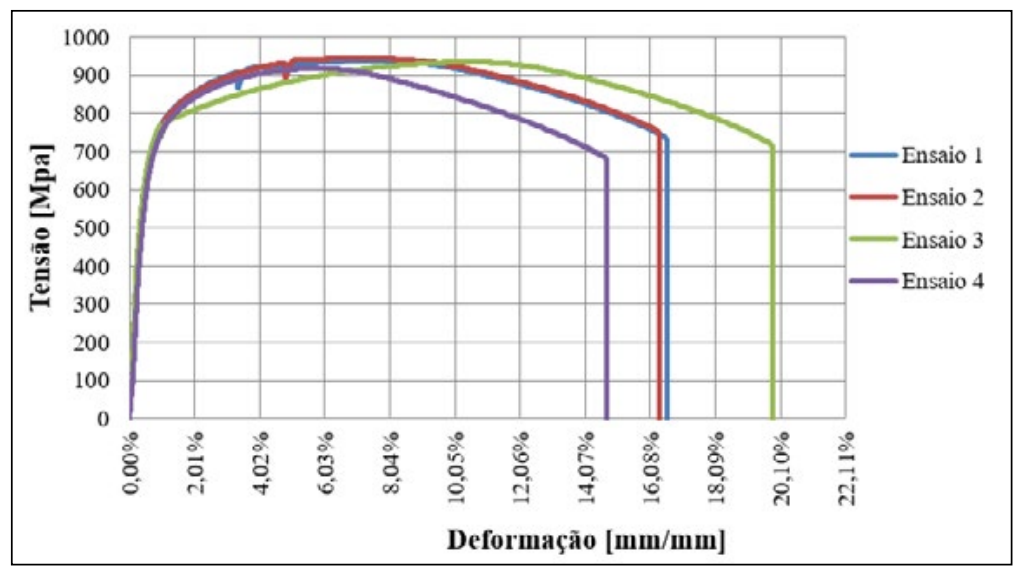

Fonte: Do autor (1017).

Para o cálculo do alongamento foi utilizada a Equação 1, após o término dos ensaios dos corpos de prova na máquina de tração. Desta forma, os resultados obtidos de alongamento para o aço 4140 estão dispostos na Tabela 4.

Tabela 4 - Alongamento para o aço 4140

\begin{tabular}{|c|c|c|c|c|c|c|}
\hline \multicolumn{7}{|c|}{ Alongamento do aço 4140} \\
\hline & \multicolumn{4}{|c|}{$\mathrm{N}^{\circ}$ do ensaio } & \multirow{2}{*}{$\begin{array}{c}\text { Valor } \\
\text { Médio }\end{array}$} & \multirow{2}{*}{$\begin{array}{l}\text { Desvio } \\
\text { Padrão } \\
\end{array}$} \\
\hline Amostras & 1 & 2 & 3 & 4 & & \\
\hline $\mathrm{Li}(\mathrm{mm})$ & 25,00 & 25,00 & 25,00 & 25,00 & 25,00 & 0,00 \\
\hline Lf (mm) & 29,15 & 29,09 & 29,96 & 29,06 & 29,31 & 0,37 \\
\hline Lf - Li (mm) & 4,15 & 4,09 & 4,96 & 4,06 & 4,31 & 0,37 \\
\hline Alongamento (\%) & 16,60 & 16,36 & 19,84 & 16,23 & 17,26 & 1,50 \\
\hline
\end{tabular}

Fonte: Do autor (2017).

Os resultados de estricção foram obtidos através da Equação 2, e dispostos na Tabela 5 . 
Tabela 5 - Estrição para o aço ASTM 4140

\begin{tabular}{|c|c|c|c|c|c|c|}
\hline \multicolumn{7}{|c|}{ Estricção do aço 4140} \\
\hline \multirow[b]{2}{*}{ Amostras } & \multicolumn{4}{|c|}{$\mathrm{N}^{\circ}$ do ensaio } & \multirow{2}{*}{$\begin{array}{c}\text { Valor } \\
\text { Médio }\end{array}$} & \multirow{2}{*}{$\begin{array}{l}\text { Desvio } \\
\text { Padrão }\end{array}$} \\
\hline & 1 & 2 & 3 & 4 & & \\
\hline $\mathrm{Di}(\mathrm{mm})$ & 6,30 & 6,30 & 6,30 & 6,23 & 6,28 & 0,00 \\
\hline Df (mm) & 4,51 & 4,36 & 4,39 & 4,35 & 4,40 & 0,06 \\
\hline $\mathrm{Ai}(\mathrm{mm})$ & 31,17 & 31,17 & 31,17 & 30,48 & 31,00 & 0,30 \\
\hline Af (mm) & 15,97 & 14,93 & 15,14 & 14,86 & 15,23 & 0,44 \\
\hline Estricção (\%) & 49,00 & 52,00 & 51,00 & 51,00 & 51,00 & 1,27 \\
\hline
\end{tabular}

Fonte: Do autor, através do Laboratório de Engenharia Mecânica UNIVATES (2017).

O valor médio para o módulo de elasticidade obtido é de aproximadamente $183 \mathrm{GPa}$, esse valor foi obtido através da inclinação da parte linear das curvas de carregamento, como pode ser observado na Figura 10.

Figura 10 - Linha de tendência para o Módulo de Elasticidade amostra 3

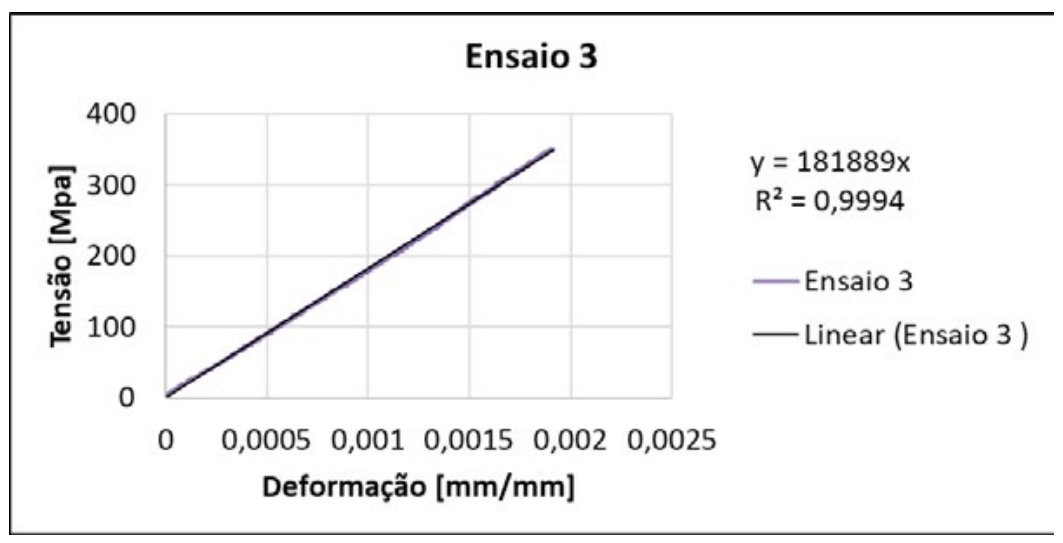

Fonte: Do autor, através do Laboratório de Engenharia Mecânica UNIVATES (2017).

Para o ensaio de impacto foram utilizados quatro corpos de prova, e o valor médio da energia de impacto foi de 18,5 Joules.

O ensaio de fadiga foi realizado pelo método Staircase. Como resultado, obteve-se um valor na faixa de 90 a $95 \mathrm{MPa}$ (limite de resistência a fadiga) para vida infinita do parafuso. Considerando uma probabilidade de $99 \%$ a máquina gerou um valor de 79,89 MPa. Quando realizado cálculos com as correções utilizando a expressão de Marin (SHIGLEY, 2011) chega-se a um valor de 97,36 MPa ligeiramente acima da faixa de valores observados na máquina de ensaios mecânicos. Essa metodologia que utiliza os fatores modificadores do limite 
de resistência à fadiga será apresentada posteriormente. Assim, na Tabela 6, podem-se observar os resultados de fadiga obtidos para o aço 4140 .

Tabela 6 - Resultados Staircase para o aço 4140

\begin{tabular}{|c|c|c|c|c|c|c|c|c|c|c|c|c|c|c|c|c|}
\hline 1 & \multicolumn{10}{|c|}{2} & 3 & 4 & 5 & 6 & 7 & 8 \\
\hline$\sigma_{\mathrm{A}}(\mathrm{MPa})$ & \multicolumn{5}{|c|}{$x$ Falha } & \multicolumn{5}{|c|}{ o Não Falha } & $x$ & 0 & $z$ & $f$ & $z f$ & $z^{2} f$ \\
\hline 105,00 & & & & & & & & & & $x$ & 1 & 0 & 3 & 1 & 3 & 9 \\
\hline 100,00 & & & & & $x$ & & & & 0 & & 1 & 1 & 2 & 1 & 2 & 4 \\
\hline 95,00 & & $\mathrm{x}$ & & 0 & & $x$ & & 0 & & & 2 & 2 & 1 & 2 & 2 & 2 \\
\hline 90,00 & 0 & & 0 & & & & 0 & & & & 0 & 3 & 0 & 0 & 0 & 0 \\
\hline Amostra $\mathrm{N}^{0}$ & 1 & 2 & 3 & 4 & 5 & 6 & 7 & 8 & 9 & 10 & & & & & & \\
\hline \multicolumn{11}{|c|}{ Somatório das colunas $3,4,6,7$ e 8} & 4 & 6 & - & 4 & 7 & 15 \\
\hline & & & & & & & & & & & & & & c & A & E \\
\hline
\end{tabular}

Fonte: Adaptado pelo autor de ISO 380 (1964).

Onde:

Coluna 1 - Amplitude da carga dinâmica aplicada;

Coluna 2 - Indicador dos eventos (falha " $x$ ", não falha "o");

Coluna 3 - Número de falhas por carga dinâmica;

Coluna 4 - Número de não falhas por carga dinâmica; dinâmica;

Coluna 5 - Ordinal " $\mathrm{z}$ ", iniciando com 0 no menor valor de carga

Coluna 6 - Repetição dos valores da coluna ( 3 ou 4) de menor soma;

Coluna 7 - Produto da coluna 5 e 6 (zf);

Coluna 8 - Produto da coluna 5 e $6\left(\mathrm{z}^{2} \mathrm{f}\right)$;

O método calcula a carga dinâmica para $50 \%$ de probabilidade de sobrevivência do componente através da Equação 3.

$$
F_{A 50}=F_{a o}+\Delta F_{\text {all }}\left(\frac{A}{c} \pm x\right)
$$

O desvio padrão de carga média de resistência é calculado através da Equação 4.

$$
S\left(F_{A}\right)=1,62 \Delta F_{\text {all }}\left(\frac{C E-A^{2}}{c^{2}}+0,029\right)
$$

$\mathrm{F}_{\mathrm{A} 50}=$ Carga dinâmica média para $50 \%$ de probabilidade de sobrevivência do componente eventos

$\mathrm{F}_{\mathrm{ao}}=$ menor carga dinâmica nas colunas 3 e 6 com um número menor de

$\Delta \mathrm{F}_{\text {all }}=$ variação da carga entre dois níveis subsequentes de carregamento $\mathrm{S}\left(\mathrm{F}_{\mathrm{A}}\right)=$ desvio padrão 
$\mathrm{C}=$ resultado do somatório da coluna 6

$\mathrm{A}=$ resultado do somatório da coluna 7

$\mathrm{E}=$ resultado do somatório da coluna 8

$\mathrm{x}$ = quando a coluna $6=$ coluna 4 (valor de $+0,5)$; quando a coluna $6=$ coluna 3 (valor de $-0,5$ )

Logo através das Equações 3 e 4 chegou-se aos seguintes resultados para $\mathrm{F}_{\mathrm{A} 50}$ e $\mathrm{S}\left(\mathrm{F}_{\mathrm{A}}\right)$ :

$$
\begin{aligned}
& \sigma_{A 50}=90+5 *\left(\frac{7}{4}+0,5\right) \\
& \sigma_{A 50}=96,25 \mathrm{MPa} \\
& S\left(F_{A}\right)=1,62 * 5 *\left(\frac{4 * 15-7^{2}}{4^{2}}+0,029\right) \\
& S\left(F_{A}\right)=5,80 \mathrm{MPa}
\end{aligned}
$$

Com os dados anteriores e considerando uma carga dinâmica de 50\% de probabilidade de sobrevivência do componente calculados, obtém-se os resultados observados na Tabela 7.

Tabela 7 - Resultados para 50\% de probabilidade para que o componente sobreviva à carga dinâmica

\begin{tabular}{c|c|c|c|c|c|c|c}
\hline Material & $\mathbf{F}_{\text {ao }}(\mathbf{M P a})$ & $\left.\Delta \mathbf{F}_{\text {all }} \mathbf{( M P a}\right)$ & $\mathbf{A}$ & $\mathbf{C}$ & $\mathbf{E}$ & $\left.\mathbf{S}\left(\mathbf{F}_{\mathbf{A}}\right) \mathbf{( M P a}\right)$ & $\mathbf{F}_{\mathrm{A} 50}(\mathbf{M P a})$ \\
\hline Aço 4140 & 90 & 5 & 7 & 4 & 15 & 5,80 & 96,25 \\
\hline
\end{tabular}

Fonte: Do autor (2017).

Através dos dados obtidos e utilizando um método estatístico, constante de Student, foi possível calcular a carga de oscilação para que o componente tenha $99 \%$ de probabilidade de sobrevivência. Para esta avaliação, utilizou-se a Equação 9.

$$
F_{A 99}=F_{A 50}-t . S\left(F_{A}\right)
$$

Onde,

$\mathrm{F}_{\mathrm{A} 99}=$ carga dinâmica para $99 \%$ de probabilidade de sobrevivência do componente;

$\mathrm{F}_{\mathrm{A} 50}=$ carga dinâmica média para $50 \%$ de probabilidade de sobrevivência do componente;

$\mathrm{t}=$ constante de student;

$\mathrm{S}\left(\mathrm{F}_{\mathrm{A}}\right)=$ desvio padrão; 
Assim, com a disposição de todos os dados, pode-se calcular utilizando a Equação 9 a carga dinâmica para $99 \%$ de sobrevivência do componente. Os resultados obtidos podem ser observados na Tabela 8 .

Tabela 8 - Resultados para $99 \%$ de probabilidade para que o componente sobreviva a carga dinâmica

\begin{tabular}{c|c|c|c|c}
\hline Material & $\mathbf{F}_{\text {A50 }}(\mathbf{M P a})$ & $\mathbf{T}$ & $\mathbf{S}\left(\mathrm{F}_{\mathbf{A}}\right)(\mathbf{M P a})$ & $\mathbf{F}_{A 99}(\mathbf{M P a})$ \\
\hline Aço $4 \mathbf{4 1 0}$ & 96,25 & 2,821 & 5,8 & 79,89 \\
\hline
\end{tabular}

Fonte: Do autor (2017).

Segundo Kloos e Thomala (1979), parafusos de aço 4140 devem romper no primeiro filete da rosca, em virtude de haver um maior concentrador de tensões nesse local. Pode-se observar na Figura 11 que realmente essa condição acontece nos parafusos ensaiados.

Figura 11 - Falha por fadiga no parafuso de aço 4140

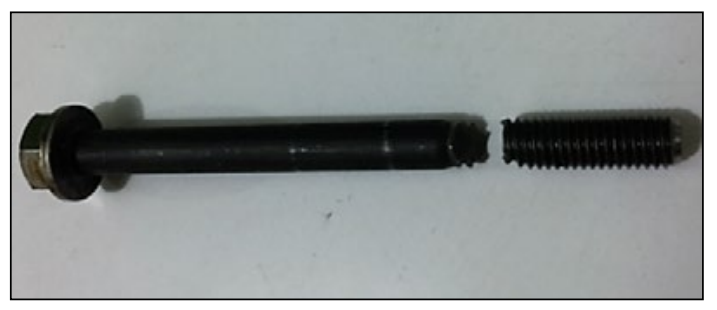

Fonte: Do autor (2017).

Bannantine, Comer e Handrock (1990), citam a utilização da Equação de Marin expressa na Equação 10 para ajustar o limite de resistência à fadiga considerando os efeitos dos parâmetros modificadores do limite de resistência a fadiga do material.

$\mathrm{S}_{\mathrm{e}}=\mathrm{K}_{\mathrm{a}} \mathrm{K}_{\mathrm{b}} \mathrm{K}_{\mathrm{c}} \mathrm{K}_{\mathrm{d}} \mathrm{K}_{\mathrm{e}} \mathrm{K}_{\mathrm{f}} \mathrm{S}_{\mathrm{e}}^{\prime}$

Onde:

$\mathrm{K}_{\mathrm{a}}$ = fator de modificação da condição de superfície;

$\mathrm{K}_{\mathrm{b}}$ = fator de tamanho;

$\mathrm{K}_{\mathrm{c}}$ = fator de carregamento;

$\mathrm{K}_{\mathrm{d}}=$ fator de temperatura;

$\mathrm{K}_{\mathrm{e}}$ = fator de confiabilidade;

$\mathrm{K}_{\mathrm{f}}=$ fator de modificação por efeitos variados;

$\mathrm{S}_{\mathrm{e}}^{\prime}=$ resistência à fadiga corrigida; 
$\mathrm{S}_{\mathrm{e}}=$ limite de resistência à fadiga no local crítico de uma peça.

Quando não há os ensaios de fadiga de peças, são realizadas estimativas utilizando os fatores modificadores de Marin, onde cada $\mathrm{K}$ representa a seguinte condição:

- Fator de superfície $\mathrm{K}_{\mathrm{a}}$.

$\mathrm{Se}^{\prime}=0,5$ Sut

$\mathrm{Se}^{\prime}=466,874 \mathrm{MPa}$

Realizando a correção para o parafuso:

- Ka (forjado)

$\mathrm{Ka}=\mathrm{a}$ Sut b (20)

$\mathrm{Ka}=0,272 \times 933,748-0,995$

$\mathrm{Ka}=0,3014$

$-\mathrm{Kb}=1$

$-\mathrm{Kc} \rightarrow$ Axial

$-K_{c}=1$

- $\mathrm{Kd}=1$ (temperatura ambiente)

- Ke $=0,814$ (confiabilidade de 99\%)

- $\mathrm{Kf}=0$ (sem acabamento)

$\mathrm{Se}=\mathrm{Ka} \mathrm{Kb} \mathrm{Kc} \mathrm{Kd} \mathrm{Ke} \mathrm{Kf} \mathrm{S}^{\prime}$

$\mathrm{Se}=(0,3014) \times(1) \times(0,85) \times(1) \times(0,814) \times(466,874)$

$\mathrm{Se}=97,36 \mathrm{MPa}$

Assim o valor 97,36 MPa representa a vida infinita do parafuso. Quando avaliadas as correções percebe-se que o valor está um pouco acima da faixa de 90 a $95 \mathrm{MPa}$ gerado pela máquina de ensaios. Após o ensaio de fadiga, observamse na Figura 12 as marcas de praia através da análise visual da fratura, onde (A) representa o início da trinca, (B) propagação da trinca e (C) ruptura total. 
Figura 12 - Análise visual da fratura obtida com microscópico manual

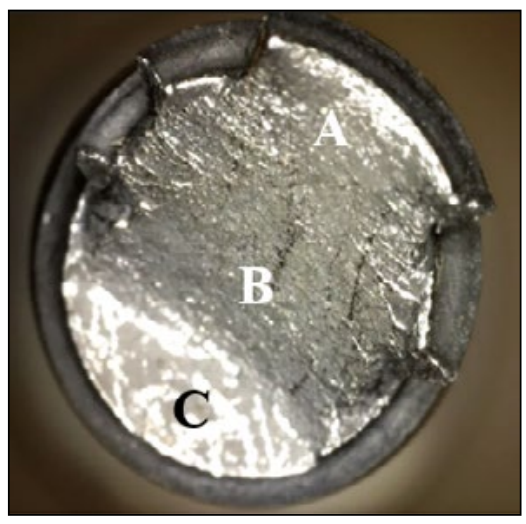

Fonte: Do autor, através do Laboratório de Engenharia Mecânica UNIVATES (2017).

A partir dos resultados anteriores, destaca-se que para o emprego adequado do material, é necessário conhecer as propriedades do material utilizado, assim como as solicitações que serão submetidas esse componente. Um resumo de todos os resultados dos ensaios mecânicos do aço 4140 é apresentado na Tabela 9.

Tabela 9 - Resultados gerais para o aço 4140

\begin{tabular}{|c|c|c|c|}
\hline \multirow{2}{*}{ Condições } & $\mathrm{T}\left({ }^{\circ} \mathrm{C}\right)=25^{\circ} \mathrm{C}$ & & \multirow{2}{*}{ Referência } \\
\hline & Normalizado e Recozido & & \\
\hline Propriedades & Valores médios & & \\
\hline Dureza (HRc) & 27,70 & Atende & 26 a 42 \\
\hline $\begin{array}{l}\text { Resistência à } \\
\text { Tração (MPa) }\end{array}$ & 933,75 & Atende & $\begin{array}{l}862 \text { Arcelormittal } \\
758 \text { Gerdau }\end{array}$ \\
\hline $\begin{array}{c}\text { Resistencia ao } \\
\text { Escoamento (MPa) }\end{array}$ & 661,58 & Atende & $\begin{array}{l}690 \text { Arcelormittal } \\
655 \text { Gerdau }\end{array}$ \\
\hline Alongamento (\%) & 17,26 & Atende & $\begin{array}{c}18 \text { a } 26 \% \\
\text { Normalizado e } \\
\text { Recozido (Shigley) }\end{array}$ \\
\hline Estricção (\%) & 51,00 & Atende & $\begin{array}{l}47 \% \text { Normalizado } \\
57 \% \text { Recozido }\end{array}$ \\
\hline $\begin{array}{c}\text { Módulo de } \\
\text { Elasticidade (MPa) }\end{array}$ & 182784 & $\begin{array}{c}\text { Não } \\
\text { atende }\end{array}$ & 190000 a 210000 \\
\hline $\begin{array}{l}\text { Resistência ao } \\
\text { Impacto }(\mathrm{J})\end{array}$ & 18,50 & - & $\begin{array}{l}23 \text { Normalizado } \\
54 \text { Recozido }\end{array}$ \\
\hline $\begin{array}{l}\text { Resistencia à } \\
\text { fadiga }(\mathrm{MPa})\end{array}$ & $\begin{array}{c}\text { vida infinita (Ensaio } 99 \% \\
\text { probabilidade igual a } 79,89 \mathrm{MPa} \text { ) }\end{array}$ & - & $\begin{array}{l}\text { Calculado igual a } \\
97,36 \mathrm{MPa}\end{array}$ \\
\hline
\end{tabular}

Fonte: Do autor (2017). 


\section{CONCLUSÃO}

É crescente o interesse pela área da mecânica na análise e caracterização de materiais devido à necessidade de seleção adequada do material baseado no desempenho do sistema em estudo. A análise e a caracterização de materiais é uma área importante que permite a seleção adequada do material baseado no desempenho do sistema em estudo assim como para o desenvolvimento de novos materiais. Com a realização padronizada dos ensaios mecânicos no aço SAE 4140, foi possível submeter os componentes fabricados as solicitações que serão aplicadas no cotidiano. Para que não haja o emprego impróprio de material ou ainda desperdício do mesmo, deve-se realizar a escolha/seleção dos materiais usados em diversas aplicações de engenharia após a realização adequada de ensaios experimentais. Através dos resultados obtidos nos ensaios mecânicos, pode-se obter a previsão da vida em fadiga de componentes mecânicos. Os resultados deste trabalho indicaram que para uma probabilidade de sobrevivência de 99 \% a carga dinâmica foi de 79,89 MPa. Respeitando esse valor para carga evitam-se consequências mais graves, como por exemplo, a quebra inesperada de um componente. Outro fator de extrema importância é o tratamento térmico do material, pois este pode alterar de maneira significativa os valores utilizados como referência em um projeto mecânico. Um exemplo disso, é o módulo de elasticidade que segundo Rocha (2004) encontra-se na faixa 190 a $210 \mathrm{GPa}$, para o aço 4140, e, neste caso, apresentou valores médios próximos a $183 \mathrm{GPa}$. No projeto mecânico, tratando-se de uma análise linear elástica, considerar um módulo de elasticidade de maior magnitude significa aumentar os níveis de tensões que irão atuar em um dado componente mecânico, para um mesmo grau de deformação, maximizando assim, os coeficientes de segurança adotados ou as dimensões do componente, ou seja, a favor da segurança. Apesar de alguns parâmetros apresentarem variações de magnitude quando comparados com os valores pesquisados na literatura, por exemplo, o valor do alongamento de $17,26 \%$ se aproxima muito do valor de referência. Destaca-se que de uma forma geral, que os resultados obtidos neste trabalho são valores consistentes, encontrando-se dentro de uma faixa aceitável de valores, por exemplo, quanto aos valores dos limites de resistência à tração e escoamento do material. São diversos os fatores que causam interferência nos resultados de ensaios experimentais, como falha do operador, incertezas sistemáticas e aleatórias, não linearidades, incertezas nos equipamentos, processos de medições e composição da microestrutura dos elementos de liga dos materiais entre outros. Esses são alguns dos parâmetros que podem ser citados, causando alterações nos valores das propriedades mecânicas avaliadas experimentalmente. 


\section{REFERÊNCIAS}

ABNT. Associação Brasileira de Normas Técnicas. NBR ISO 6892. Materiais metálicos- Ensaios de tração à temperatura ambiente. Rio de Janeiro: ABNT, 2002.

. NBR NM 87: Aço carbono e ligados para construção mecânica - Designação e composição química. Rio de Janeiro: ABNT, 2000.

ABRAHÃO, R.R.R. et al. Fadiga de materiais: uma revisão bibliográfica. In: ENCONTRO INTERNO, 8; SEMINÁRIO DE INICIAÇÃO CIENTÍFICA, 12, Anais... Uberlândia: UFU, 2008. Disponível em: <https:/ / ssl4799.websiteseguro.com/swge5/ seg/cd2008/PDF/IC2008-0165.PDF>. Acesso em: 20 ago. 2016.

ALVARENGA JÚNIOR, Álvaro; MANSUR, Tanius R.; PALMA, Ernani S. Influência da refrigeração e da sequência de aplicação de cargas em ensaios de fadiga flexorotativos nas propriedades mecânicas do aço SAE 8620. In: CONGRESSO NACIONAL DE ENGENHARIA MECÂNICA, 2, Anais... João Pessoa, 2002

ASTM. American Society for Testing and Materials. ASTM 4140: Hot Rolled High Tensile Steel Bar. [S.1.]: ATSM, 2013.

ASTM E23: Standard Test Methods for Notched Bar Impact Testing of Metallic Material. [S.1.]: ASTM, 2002.

AFZAL, A.; FATEMI, A. A Comparative Study of Fatigue Behavior and Life Predictions of Forged Steel and PM Connecting Rods. SAE Technical Paper, v. 1, n. 1529, 2004. Disponível em: <http://papers.sae.org/2004-01-1529/>. Acesso em: 20 ago. 2016.

BANNANTINE, J. A.; COMER, J.; HANDROCK, J. Fundamentals of fatigue anlysis. New Jersey: Prentice Hall, 1990.

CALLISTER JR, Willian D. Ciência e engenharia de materiais: uma introdução. 5. ed. Rio de Janeiro: LTC, 2002.

CHIAVERINI, Vicente. Aços e ferros fundidos. 7. ed. São Paulo: ABM, 2005.

DALCIN, Gabrieli B. Ensaios de materiais. 2007. 41f. Monografia (Graduação em Engenharia Industrial Mecânica) - Universidade Regional Integrada do Alto Uruguai e das Missões, Santo Ângelo, 2007. Disponível em: <http:/ /www.urisan.tche. br/ lemm/arquivos/ensaios_mecanicos.pdf>. Acesso em: 20 ago. 2016.

DOWLING, Norman E. Mechanical behavior of materials. 2. ed. New Jersey: McGraw- Hill, 1999.

EMPRESA METALMECÂNICA. 2017. [arquivo particular]. 
EUROPEAN STANDARD. Norma DIN 10083: Steels for quenching and tempering part 2: Technical delivery conditions for non alloy steels. Deutsche: European Standard, 2006.

Norma DIN EN 1665: Sechskantschrauben mit Flansch schwere Reihe Deutsche Fassung. Deutsche: European Standard, 1998.

GRIZA, Sandro. Efeito do torque na vida em fadiga de uniões parafusadas. 2000. 107f. Dissertação (Mestrado em Engenharia) Universidade Federal do Rio Grande do Sul, Porto Alegre, 2000. Disponível em: <http:/ / www.lume.ufrgs.br/ handle/10183/14382>. Acesso em: 20 ago. 2016.

ISO. International Organization for Standardization. ISO 380: Sports and other recreational facilities and equipment. Withdrawn: ISO, 1964.

KLOOS, K. H., THOMALA, W. Spannungsverteilung im Schraubengewinde. VDIZ, v. 121, n. 12, 1979.

LABORATÓRIO DA UFRGS. 2017. [arquivo particular].

LABORATÓRIO DE ENGENHARIA MECÂNICA UNIVATES. 2017. [arquivo particular].

MARUTA, S. et al. A Study on High Cycle Fatigue Behavior in Lead. In: INTERNATIONAL CONFERENCE ON FRACTURE \& STRENGTH OF SOLIDS, 9. Anais... Korea, 2013. Disponível em: <https: / / www.researchgate.net/ publication/281594176_A_Study_on_High_Cycle_Fatigue_Behavior_in_Lead >. Acesso em: 31 ago. 2016.

MATLOCK, David K. et al. Surface processing to improve the fatigue resistance of advanced bar steels for automotive applications. Mat. Res., v. 8, n. 4, p. 453-459, 2005. Disponível em: <http:/ / www.scielo.br/scielo.php?script=sci_ arttext\&pid=S1516-14392005000400017> . Acesso em: 20 ago. 2016.

NORTON, Robert L. Projeto de máquinas. São Paulo: Bookman, 2013.

RAGAGNIN, Luido Tolfo. Avaliação de fadiga em haste de compressor alternativo. 2015. 27f. Trabalho de Conclusão (Graduação em Engenharia Mecânica) Universidade Federal do Rio Grande do Sul, Porto Alegre, 2015. Disponível em: <http:/ / www.lume.ufrgs.br/handle/10183/131313>. Acesso em: 20 ago. 2016.

ROCHA, M. A. C. Análise das propriedades Mecânicas do aço SAE 4140 com estrutura bifásica. 2004. 10f. Artigo (Mestrado em Engenharia Mecânica) Universidade Federal Fluminense, Niterói, 2004.

SENA, Betânia M. Avaliação da resistência a fadiga de um aço DP-780 de aplicação automotiva por intermédio de ensaios com controle de tensão. 2010. 65f. Monografia (Graduação em Engenharia Metalúrgica) - Universidade Federal de Ouro Preto, Ouro Preto, 2010. Disponível em: <http:/ / www.em.ufop.br/demet/download/mo- 
nografias / AVALIACAODARESISTENCIAAFADIGADEUMACODP780DEAPLICACAOAUTOMOTIVAPORINTERMEDIODEENSAIOSCOMCONTROLEDETENSAO. pdf $>$. Acesso em: 20 ago. 2016.

SHIGLEY, Joseph E.; BUDYNAS, Richard G.; NISBETT, J Keith. Elementos de máquina de Shigley. 8. ed. São Paulo: McGraw-Hill, 2011.

TOSO, Marcelo A. et al. Ensaio de fadiga: um comparativo entre dois corpos de provas distintos para aplicação em molas de suspensão automotiva. In: CONGRESSO NACIONAL DE ENGENHARIA MECÂNICA, 9, 2016. Anais... Fortaleza: ABCM, ago. 2016. 